\title{
POLYNOMIALS RELATED TO THE BESSEL FUNCTIONS
}

\section{BY}

F. T. HOWARD

ABSTRACT. In this paper we examine the polynomials $w_{n}(a)$ defined by means of

$$
-4 e^{x a}\left[x\left(e^{x}-1\right)-2\left(e^{x}+1\right)\right]^{-1}=\sum_{n=0}^{\infty} w_{n}(a) x^{n} / n ! .
$$

These polynomials are closely related to the zeros of the Bessel function of the first kind of index $-3 / 2$, and they are in some ways analogous to the Bernoulli and Euler polynomials. This analogy is discussed, and the real and complex roots of $w_{n}(a)$ are investigated. We show that if $n$ is even then $w_{n}(a)>0$ for all $a$, and if $n$ is odd then $W_{n}(a)$ has only the one real root $a=1 / 2$. Also we find upper and lower bounds for all $b$ such that $w_{n}(a+b i)=0$. The problem of multiple roots is discussed and we show that if $n \equiv 0,1,5,8$ or $9(\bmod 12)$, then $w_{n}(a)$ has no multiple roots. Finally, if $n \equiv 0,1,2,5,6$ or $8(\bmod 12)$, then $W_{n}(a)$ has no factor of the form $a^{2}+c a+d$ where $c$ and $d$ are integers.

1. Introduction. The purpose of this paper is to investigate the polynomials $W_{n}(a)$ defined by means of

$$
\frac{-4 e^{x a}}{x\left(e^{x}-1\right)-2\left(e^{x}+1\right)}=\sum_{n=0}^{\infty} w_{n}(a) \frac{x^{n}}{n !} .
$$

These polynomials, first introduced in [7], are of interest because of their relationship to the zeros of the Bessel function and because they are in some ways analogous to the well-known Bernoulli and Euler polynomials. Throughout this paper we let $W_{n}=W_{n}(0)$.

The relationship to the Bessel function of the first kind, $J_{\nu}(z)$, is found by letting

$$
\sigma_{2 n}(\nu)=\sum_{m=1}^{\infty}\left(j_{\nu, m}\right)^{-2 n} \quad(n=1,2, \ldots),
$$

where the $j_{\nu, m}$ are the zeros of $z^{-\nu} J_{\nu}(z)$. Then, as pointed out in [7],

$$
\sigma_{2 n}(-3 / 2)=(-1)^{n} 2^{2 n-3} W_{2 n-2} /(2 n-2) ! .
$$

Received by the editors July 24, 1974.

AMS (MOS) subject classifications (1970). Primary 10A40; Secondary 12D05, 12D10, 33A40, 05A10.

Key words and phrases. Rayleigh function, Bernoulli polynomial, Euler polynomial, van der Pol polynomial, Bessel polynomials, polynomials (mod 2).

Copyright $\odot$ 1975, American Mathematical Society 
This relationship will be more fully discussed in $\S 5$, where we show how the generating functions for the Euler and Bernoulli polynomials can be generalized so as to produce numbers analogous to $W_{n}$ and formulas similar to (1.3), i.e., formulas involving $\sigma_{2 n}(\nu)$ where $\nu$ is half an odd integer. It should be mentioned that the function $\sigma_{2 n}(\nu)$, known as the Rayleigh function, has been the subject of a number of investigations. Its early history can be found in [22, p. 502], and more recent papers include [3], [4], [10]-[13] and [15]-[17].

Some of the results concerning $W_{n}(a)$ which are proved in this paper are:

(a) $W_{2 n}(a)>0$ for all real $a$.

(b) $W_{2 n+1}(a)$ has only one real root $a=1 / 2$.

(c) If $2 b^{2}<1$ and $a \neq 1 / 2$ then $W_{n}(a+b i) \neq 0$.

(d) If $0<r b^{2}<1$, where $r=2$ if $n$ is odd and $r=6$ if $n$ is even, then $W_{n}(1 / 2+b i) \neq 0$.

(e) If $4 b^{2}>m(m+1)$ and $0 \leqslant a \leqslant 1$, then $W_{m}(a+b i) \neq 0$.

(f) If $n>0$ then

$$
n ! 2^{-n} 3^{-[(n+1) / 2]}<\left|W_{n}\right|<n ! 2^{-n+1} .
$$

(g) If $n \equiv 0,1,5,8$ or $9(\bmod 12)$, then $W_{n}(a)$ has no multiple roots.

(h) If $n \equiv 0,1,2,5,6$ or $8(\bmod 12)$, then $W_{n}(a)$ has no factor of the form $a^{2}+c a+d$ when $c$ and $d$ are integers.

2. Basic properties of $W_{n}(a)$. The polynomials $W_{n}(a)$ we are discussing in this paper are the same as the polynomials $C_{n}(a)$ defined in [7]. The notation has been changed to avoid confusion with the numbers $C_{n}$ discussed in $[19, \mathrm{p}$. 27].

In [7] it is proved that

$$
\begin{gathered}
W_{2 n}=-n W_{2 n-1}>0 \quad(n>0), \\
W_{n}(1)=(-1)^{n} W_{n},
\end{gathered}
$$

and the following formulas are derived:

$(2.3)(n+1) W_{n}(a+1)-(n+1) W_{n}(a)-2 W_{n+1}(a+1)-2 W_{n+1}(a)=-4 a^{n+1}$,

$$
2(2 n+1) W_{2 n}=2+\sum_{r=0}^{n-1}\left(\begin{array}{c}
2 n+1 \\
2 r
\end{array}\right)(2 n-2 r-1) W_{2 r}
$$

It also follows from (1.1) that for $n>0$

$$
4 W_{n}(a)=4 a^{n}-2 n W_{n-1}(a)+\sum_{r=0}^{n-2}\left(\begin{array}{l}
n \\
r
\end{array}\right)(n-r-2) W_{r}(a) .
$$

It follows from (2.4) that 


$$
p W_{n} \equiv 0(\bmod p)
$$

if $p$ is an odd prime. That is, if $W_{n}$ is reduced to its lowest terms, the only possible prime dividing its denominator is 2 . In fact, by a theorem in [5] we have

$$
2^{t} W_{2 n} \equiv 1(\bmod 2) \text { if } 2^{t} \|_{n}+1 \text {. }
$$

Here the notation $2^{t} \| n+1$ means $2^{t}$ is the highest power of 2 dividing $n+1$. Thus by (2.1) we have for $n$ odd,

$$
2^{t} W_{2 n-1} \equiv 1(\bmod 2) \text { if } 2^{t} \| n+1,
$$

and if $n$ is even

$$
2^{t} W_{2 n-1} \equiv 1(\bmod 2) \text { if } 2^{t} \| n .
$$

Note that by (2.6) $W_{2 n}$ is an odd integer if $n$ is even.

By (1.1) we see that

$$
\frac{-4}{x\left(e^{x}-1\right)-2\left(e^{x}+1\right)}=\sum_{n=0}^{\infty} w_{n} \frac{x^{n}}{n !} .
$$

It follows from (2.4) that $w_{0}=1, w_{1}=-1 / 2, w_{2}=1 / 2, W_{3}=-1 / 2, w_{4}=$ $1, W_{5}=-7 / 4$. The first 15 values of $W_{n}$ are listed in [7], though there is a misprint involving $W_{13}$ and $W_{14}$. The correct values are

$$
\begin{array}{ll}
W_{13}=-660,651 / 8, & W_{15}=-24,099,103 / 8, \\
W_{14}=4,624,557 / 8, & W_{16}=24,099,103 .
\end{array}
$$

The first 9 polynomials are

$$
\begin{aligned}
& W_{0}(a)=1 \\
& W_{1}(a)=a-\frac{1}{2} \\
& W_{2}(a)=a^{2}-a+\frac{1}{2} \\
& W_{3}(a)=a^{3}-\frac{3}{2} a^{2}+\frac{3}{2} a-\frac{1}{2} \\
& W_{4}(a)=a^{4}-2 a^{3}+3 a^{2}-2 a+1, \\
& W_{5}(a)=a^{5}-\frac{5}{2} a^{4}+5 a^{3}-5 a^{2}+5 a-\frac{7}{4}, \\
& W_{6}(a)=a^{6}-3 a^{5}+\frac{15}{2} a^{4}-10 a^{3}+15 a^{2}-\frac{21}{2} a+\frac{21}{4}, \\
& W_{7}(a)=a^{7}-\frac{7}{2} a^{6}+\frac{21}{2} a^{5}-\frac{35}{2} a^{4}+35 a^{3}-\frac{147}{4} a^{2}+\frac{147}{4} a-\frac{51}{4}, \\
& W_{8}(a)=a^{8}-4 a^{7}+14 a^{6}-28 a^{5}+70 a^{4}-98 a^{3}+147 a^{2}-102 a+51 .
\end{aligned}
$$


The roots of $W_{2}(a)$ are $1 / 2 \pm 1 / 2 i$ and the roots of $W_{3}(a)$ are $1 / 2,1 / 2 \pm i \sqrt{3} / 2$. It follows from (1.1) that

$$
W_{n}(a)=\sum_{r=0}^{n}\left(\begin{array}{l}
n \\
r
\end{array}\right) W_{n-r} a^{r}=\sum_{r=0}^{n}\left(\begin{array}{l}
n \\
r
\end{array}\right) w_{r} a^{n-r},
$$

so

$$
W_{n}^{\prime}(a)=n W_{n-1}(a),
$$

and

$$
\begin{aligned}
W_{2 n}(a+b i)= & \sum_{r=0}^{n}\left(\begin{array}{c}
2 n \\
2 r
\end{array}\right) b^{2 n-2 r}(-1)^{n-r} W_{2 r}(a) \\
& +i \sum_{r=0}^{n-1}\left(\begin{array}{c}
2 n \\
2 r+1
\end{array}\right) b^{2 n-2 r-1}(-1)^{n-r} W_{2 r+1}(a), \\
W_{2 n+1}(a+b i)= & \sum_{r=0}^{n}\left(\begin{array}{c}
2 n+1 \\
2 r+1
\end{array}\right)(-1)^{n-r} b^{2 n-2 r} W_{2 r+1}(a) \\
& +i \sum_{r=0}^{n}\left(\begin{array}{c}
2 n+1 \\
2 r
\end{array}\right)(-1)^{n-r} b^{2 n+1-2 r} W_{2 r}(a) .
\end{aligned}
$$

Relationships to the Bernoulli and Euler polynomials can be derived from (1.1). The Euler polynomials $E_{n}(a)$ are defined by

$$
\frac{2 e^{a x}}{e^{x}+1}=\sum_{n=0}^{\infty} E_{n}(a) \frac{x^{n}}{n !}
$$

Thus we have

$$
\begin{aligned}
\sum_{n=0}^{\infty} W_{n}(a) \frac{x^{n}}{n !} & =\frac{2 e^{a x}}{e^{x}+1} \cdot \frac{-4\left(e^{x}+1\right)}{2 x\left(e^{x}-1\right)-2\left(e^{x}+1\right)} \\
& =\sum_{n=0}^{\infty} E_{n}(a) \frac{x^{n}}{n !} \cdot \sum_{n=0}^{\infty} W_{2 n} \frac{x^{2 n}}{(2 n) !}
\end{aligned}
$$

and so

$$
W_{n}(a)=\sum_{2 k<n}\left(\begin{array}{c}
n \\
2 k
\end{array}\right) W_{2 k} \cdot E_{2 n-2 k}(a) .
$$

Similarly

$$
E_{n}(a)=W_{n}(a)+\frac{n}{2} \sum_{2 k+2<n}\left(\begin{array}{c}
n-1 \\
2 k+1
\end{array}\right) E_{2 k+1}(0) W_{n-2 k-2}(a) .
$$

The Bernoulli polynomials $B_{n}(a)$ and Bernoulli numbers $B_{n}=B_{n}(0)$ are defined by 


$$
\frac{x e^{x a}}{e^{x}-1}=\sum_{n=0}^{\infty} B_{n}(a) \frac{x^{n}}{n !}
$$

Thus by (1.1) we have

$$
\begin{gathered}
(2 n+1) W_{2 n}(a)=-2 \sum_{2 k<2 n}\left(\begin{array}{c}
2 n+1 \\
2 k+1
\end{array}\right) W_{2 k+1} B_{2 n-2 k}(a), \\
B_{n}(a)=\frac{1}{2}\left(\begin{array}{l}
n \\
2
\end{array}\right) W_{n-2}(a)+\sum_{2 k \leqslant n}\left(\begin{array}{c}
n \\
2 k
\end{array}\right) B_{2 k} W_{n-2 k}(a) .
\end{gathered}
$$

The basic properties of the Euler and Bernoulli polynomials are discussed in [19, Chapter 2].

In determining whether or not $W_{n}(a)$ has multiple roots, we will need the following rule [9] for finding the highest power of 2 dividing the binomial coefficient $\left(\begin{array}{l}n \\ r\end{array}\right)$ :

LEMMA 2.1. Write $n=n_{0}+n_{1} 2+\cdots+n_{k} 2^{k}, n_{i}=0$ or $1, r=r_{0}+$ $r_{1} 2+\cdots+r_{k} 2^{k}, r_{i}=0$ or 1 . Start with the smallest $i$ such that $r_{i}>n_{i}$ and count the number of adjacent digits $r_{i}, r_{i+1}, \cdots$ such that $r_{i+j} \geqslant n_{i+j}$. Stop counting when $r_{i+j}<n_{i+j}$, resume counting when $r_{i+j}>n_{i+j}$ and repeat the process. The total number of digits counted is the highest power of 2 which divides $\left(\begin{array}{l}n \\ r\end{array}\right)$.

3. Inequalities and the real roots of $W_{n}(a)$. We begin by showing that $W_{2 n}(a)$ has no real roots and $W_{2 n+1}(a)$ has exactly one real root, namely $a=1 / 2$.

THEOREM 3.1. For $n \geqslant 0, W_{n}(a)=(-1)^{n} W_{n}(1-a)$.

Proof. Replacing $x$ by $-x$ in (1.1) and simplifying, we have

$$
\sum_{n=0}^{\infty} W_{n}(a)(-1)^{n} \frac{x^{n}}{n !}=\frac{-4 e^{x(1-a)}}{x\left(e^{x}-1\right)-2\left(e^{x}+1\right)}=\sum_{n=0}^{\infty} W_{n}(1-a) \frac{x^{n}}{n !} .
$$

COROLlARY. If $n$ is odd, $1 / 2$ is a root of $W_{n}(a)$.

THEOREM 3.2. For all real $a, W_{2 n}(a)>0$.

Proof. By (2.11) and (2.1) we have

$$
W_{2 n}(a)=a^{2 n}+\sum_{r=1}^{n}\left[\left(\begin{array}{c}
2 n \\
2 r
\end{array}\right) a^{2 n-2 r}-\frac{1}{r}\left(\begin{array}{c}
2 n \\
2 r-1
\end{array}\right) a^{2 n-2 r+1}\right] W_{2 r}
$$

and it is clear that if $a \leqslant 0, W_{2 n}(a)>0$. Assume $0<a \leqslant 1 / 2$. Then

$$
W_{2 n}(a) \geqslant a^{2 n}+\sum_{r=1}^{n} a^{2 n-2 r}\left[\left(\begin{array}{l}
2 n \\
2 r
\end{array}\right)-\frac{1}{2 r}\left(\begin{array}{c}
2 n \\
2 r-1
\end{array}\right)\right] W_{2 r} \text {. }
$$


Now since $\left(\begin{array}{l}2 n \\ 2 r\end{array}\right) \geqslant(2 r)^{-1}\left(\begin{array}{c}2 n \\ 2 r-1\end{array}\right), r=1, \ldots, n$, we have $W_{2 n}(a)>0$ if $-\infty<a \leqslant 1 / 2$. By Theorem 3.1, the theorem is true for all real $a$.

THEOREM 3.3. For $n \geqslant 0, w_{2 n+1}(a)$ has exactly one real root, $a=1 / 2$.

PROOF. By Theorem 3.2 and (2.12), $W_{2 n+1}(a)$ is an increasing function.

The graphs of $W_{2 n}(a)$ and $W_{2 n+1}(a)$ for $n>0$ are given in Figures 1 and 2 respectively. Note that $W_{2 n}(a)$ has its minimum value at $a=1 / 2$.

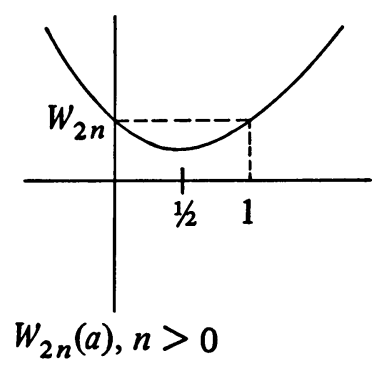

FIGURE 1

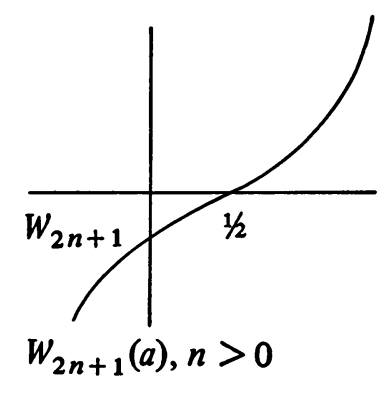

FIGURE 2

Next we prove two inequalities involving $W_{n}(a)$.

THEOREM 3.4. For all real $a$,

$$
12\left|W_{n}(a)\right| / n !>\left|W_{n-2}(a)\right| /(n-2) !
$$

except when $n$ is odd and $a=1 / 2$.

Proof. By (2.5) and the fact that $W_{2 n}\left(\frac{1}{2}\right)>0$ for all $n$, it is clear that

$$
12 W_{2 n}(1 / 2) /(2 n) !>W_{2 n-2}(1 / 2) /(2 n-2) !
$$

Also, by (2.4) it is clear that the theorem is true when $n$ is even and $a=0$ or 1 . Hence by (2.1) the theorem is true when $a=0$ or 1 for all $n$. We now proceed by induction on $n$. The theorem is true for $n=2$; assume it is true for $2, \ldots, n-1$. If $12 W_{n}(a) / n !=W_{n-2}(a) /(n-2)$ ! for some $a>1 / 2$, it follows that

$$
12 W_{n}^{\prime}(b) / n ! \leqslant W_{n-2}^{\prime}(b) /(n-2) !
$$

for some $b \geqslant 1 / 2(b>1 / 2$ if $n$ is even). Then by (2.12),

$$
12 W_{n-1}(b) /(n-1) ! \leqslant W_{n-3}(b) /(n-3) !,
$$

a contradiction.

THEOREM 3.5. For $0 \leqslant a \leqslant 1$ and $n>2$,

$$
4\left|W_{n}(a)\right| / n !<\left|W_{n-2}(a)\right| /(n-2) !
$$


Proof. We first prove the theorem for $n$ even and $a=1 / 2$. By (2.5) we

have

(3.1) $\frac{4 W_{2 n}(1 / 2)}{(2 n) !}-\frac{W_{2 n-2}(1 / 2)}{(2 n-2) !}=\frac{2^{2-2 n}}{(2 n) !}+\sum_{r=0}^{n-2} \frac{2 n-2 r-2}{(2 n-2 r) !} \frac{W_{2 r}(1 / 2)}{(2 r) !}-\frac{W_{2 n-2}\left(\frac{1}{2}\right)}{(2 n-2) !} \cdot$

Also from (2.5), replacing $n$ by $2 n-1$, we have

$$
\frac{W_{2 n-2}\left(\frac{1}{2}\right)}{(2 n-2) !}=\frac{2^{2-2 n}}{(2 n-1) !}+\frac{1}{2} \sum_{r=0}^{n-2} \frac{2 n-2 r-3}{(2 n-2 r-1) !} \frac{W_{2 r}\left(\frac{1}{2}\right)}{(2 r) !}
$$

Substituting this expression for $W_{2 n-2}(1 / 2) /(2 n-2)$ ! into the right-hand side of (3.1), we see that $4 W_{2 n}(1 / 2) /(2 n) !<W_{2 n-2}(1 / 2) /(2 n-2)$ !. Next we show the theorem is true when $a=0$ or 1 . From (2.5) we have

$$
4 W_{2 n+1}=\sum_{r=0}^{2 n-2}\left(\begin{array}{c}
2 n+1 \\
r
\end{array}\right)(2 n-1-r) W_{r}-2(2 n+1) W_{2 n},
$$

and so by (2.1),

$$
\frac{4 W_{2 n+2}}{(2 n+2) !}=\frac{W_{2 n}}{(2 n) !}-\frac{1}{2} \sum_{r=0}^{n-1} \frac{2 n-1-2 r}{(2 n+1-2 r) !} \frac{W_{2 r}}{(2 r) !}+\sum_{r=1}^{n-1} \frac{2 n-2 r}{(2 n-2 r+2) !} \frac{W_{2 r}}{(2 r) !} .
$$

Now since $(2 n-1-2 r) / 2(2 n+1-2 r) ! \geqslant(2 n-2 r) /(2 n-2 r+2) !$ for $r=1, \ldots$, $n-1$, we see that for $n \geqslant 1$

$$
4 W_{2 n+2} /(2 n+2) !<W_{2 n} /(2 n) !,
$$

and so by (2.1)

$$
4\left|W_{n}\right| / n !<\left|W_{n-2}\right| /(n-2) !
$$

for $n>2$. We now proceed by induction as we did in Theorem 3.4 to finish the proof.

COROLLARY. For $n>0$

$$
\begin{aligned}
2^{-2 n} 3^{-n}(2 n) ! & <W_{2 n}<2^{1-2 n}(2 n) !, \\
-2^{1-2 n} 3^{-n}(2 n-1) ! & >W_{2 n-1}>-2^{2-2 n}(2 n-1) !, \\
2 \cdot 3^{-n} & <\left|\sigma_{2 n+2}(-3 / 2)\right|<1 .
\end{aligned}
$$

4. Complex roots of $W_{n}(a)$.

THEOREM 4.1. If $a \neq 1 / 2$ and $2 b^{2}<1, W_{n}(a+b i) \neq 0$.

PROOF. For $W_{2 n}(a+b i)$, we examine the imaginary part of (2.13). By Theorem 3.4 the imaginary part of $(2.13)$ is never zero if $2 b^{2}<1$. For 
$W_{2 n+1}(a+b i)$ we examine the imaginary part of (2.14) and again use Theorem 3.4 to show it is never zero.

THEOREM 4.2. If $a=1 / 2$ and $0<2 b^{2}<1$, then $W_{2 n+1}(a+b i) \neq 0$. If $a=1 / 2$ and $6 b^{2}<1$, then $W_{2 n}(a+b i) \neq 0$.

PROOF. The proof for $W_{2 n+1}(a+b i)$ is the same as the proof of Theorem 4.1. Since $W_{r}(1 / 2)=0$ if $r$ is odd, we must examine the real part of (2.13) for $W_{2 n}(a+b i)$. By Theorem 3.4, the real part is never zero if $6 b^{2}<1$.

THEOREM 4.3. If $0 \leqslant a \leqslant 1$ and $4 b^{2}>m(m+1)$, then $W_{m}(a+b i) \neq 0$.

Proof. For $W_{2 n}(a+b i)$ we use Theorem 3.5 to show that the imaginary part of (2.13) is never zero. For $W_{2 n+1}(a+b i)$ we use Theorem 3.5 to show that the real part of (2.14) is never zero.

When examining $W_{n}(a)$ for multiple roots, we need to work with $W_{n}(a)$ modulo 2. The next theorem shows that if $2^{m} W_{n}$ is integral modulo 2 then $2^{m} W_{n}(a)$ has integer coefficients.

THEOREM 4.4.(a) If $n \equiv 0(\bmod 4)$ then $W_{n}(a)$ has integer coefficients. Also

$$
W_{n}(a) \equiv(1+a)^{n+2}+a^{n+2}(\bmod 2) \text {. }
$$

(b) If $n \equiv 1(\bmod 4)$ and $2^{t} \| n+3$, then $2^{t-1} W_{n}(a)$ has integer coefficients. Also

$$
2^{t-1} W_{n}(a) \equiv(1+a)^{n+3}+a^{n+3}(\bmod 2) .
$$

(c) If $n \equiv 2(\bmod 4)$ and $2^{t} \| n+2$, then $2^{t-1} W_{n}(a)$ has integer coefficients. Also

$$
2^{t-1} W_{n}(a) \equiv(1+a)^{n+2}+a^{n+2}(\bmod 2) .
$$

(d) If $n \equiv 3(\bmod 4)$ and $2^{t} \| n+1$, then $2^{t-1} W_{n}(a)$ has integer coefficients. Also

$$
2^{t-1} W_{n}(a) \equiv\left(1+a+a^{2}\right)\left[(1+a)^{n+1}+a^{n+1}\right](\bmod 2) .
$$

Proof. To prove this theorem we examine (2.11), using (2.7), (2.8), (2.9) and Lemma 2.1. We will show how to prove (d), which is the most difficult of the four cases. Suppose

$$
n+1=2^{t}+2^{t_{1}}+\cdots+2^{t_{m}}, \quad 2 \leqslant t<t_{1}<\cdots<t_{m} .
$$

By considering the different cases, namely $r=0,1,2$ or $3(\bmod 4)$, and by using Lemma 2.1, we can show that $2^{t-1}\left(\begin{array}{l}n \\ r\end{array}\right) W_{n-r} \equiv 0(\bmod 2)$ if and only if for $0 \leqslant r \leqslant$ $n$ either

$$
r=2^{u_{1}}+\cdots+2^{u_{k}},
$$




$$
\begin{aligned}
& r=1+2^{u_{1}}+\cdots+2^{u_{k}}, \\
& r=2+2^{u_{1}}+\cdots+2^{u_{k}},
\end{aligned}
$$

where $\left\{u_{1}, \ldots, u_{k}\right\}$ is any subset of $\left\{t, t_{1}, \ldots, t_{m}\right\}$. For example, suppose $r \equiv 0$ $(\bmod 4)$. If $r$ is of the form (4.1), then $n-r \equiv 3(\bmod 4)$ and

$$
n-r=-1+2^{h}+2^{h_{1}}+\cdots+2^{h_{q}} \quad\left(2 \leqslant h<h_{1} \cdots\right)
$$

where $\left\{h, h_{1}, \ldots, h_{q}\right\}$ is a subset of $\left\{t, t_{1}, \ldots, t_{m}\right\}$. By Lemma $2.1,2^{h-t} \|\left(\begin{array}{l}n \\ r\end{array}\right)$ and so $2^{t-1}\left(\begin{array}{l}n \\ r\end{array}\right) W_{n-r} \equiv 1(\bmod 2)$. If $r \equiv 0(\bmod 4)$ but $r$ is not of the form $(4.1)$, then using the same type of reasoning we see that $2^{t-1}\left(\begin{array}{c}n \\ r\end{array}\right) W_{n-r} \equiv 0(\bmod 2)$. The cases $r=1,2$, or $3(\bmod 4)$ are treated in a similar way. Thus we have, letting $t_{0}=t$,

$$
\begin{aligned}
2^{t-1} W_{n}(a) & \equiv\left(1+a+a^{2}\right) \prod_{i=0}^{m}\left(1+a^{2^{t_{i}}}\right)+a^{n+1}+a^{n+2}+a^{n+3} \\
& \equiv\left(1+a+a^{2}\right)\left((1+a)^{n+1}+a^{n+1}\right)(\bmod 2) .
\end{aligned}
$$

THEOREM 4.5. If $n \equiv 1(\bmod 4)$ then $W_{n}(a)$ has no multiple roots.

Proof. Let $d(a)$ be the unique, monic, rational coefficient GCD of $W_{n}(a)$ and $W_{n}^{\prime}(a)=n W_{n-1}(a)$. Since $n-1 \equiv 0(\bmod 4)$ it follows that $W_{n-1}(a)$ has integer coefficients, and

$$
W_{n-1}(a)=d(a) \cdot f(a)
$$

where $f(a)$ is monic with rational coefficients. By Gauss' lemma, $d(a)$ and $f(a)$ have integer coefficients. Thus, if $2^{t} \| n+3$, we can write

$$
2^{t-1} W_{n}(a)=d(a) \cdot g(a)
$$

where $g(a)$ has integer coefficients. Let $d(a) \equiv d^{*}(a)(\bmod 2)$ where the coefficients of $d^{*}(a)$ are 0 or 1 . Now since $d(a)$ divides $W_{n-1}(a)$ and $2^{t-1} W_{n}(a)$, it follows from (4.4), (4.5) and Theorem 4.4 that $d^{*}(a)$ divides $(\bmod 2)$ both $(1+a)^{n+3}+a^{n+3}$ and $(1+a)^{n+1}+a^{n+1}$. This implies that $d^{*}(a)$ divides

$$
\begin{aligned}
{\left[\left(1+a^{2}\right)\right.} & \left.+a^{2}\right]\left[(1+a)^{n+1}+a^{n+1}\right]+(1+a)^{n+3}+a^{n+3} \\
& \equiv(1+a)^{2} a^{n+1}+a^{2}(1+a)^{n+1} \\
& \equiv a^{2}(1+a)^{2}\left[(1+a)^{n-1}+a^{n-1}\right](\bmod 2) .
\end{aligned}
$$

Now if $d^{*}(a)$ divides $a^{2}$ or $(1+a)^{2}(\bmod 2)$ then $d^{*}(a)=1$, because $W_{n-1}(0) \equiv$ $W_{n-1}(1) \equiv 1(\bmod 2)$. If $d^{*}(a)$ divides $(1+a)^{n-1}+a^{n-1}(\bmod 2)$ then, as above, it follows that $d^{*}(a)$ divides $(1+a)^{n-3}+a^{n-3}(\bmod 2)$ and hence $d^{*}(a)$ divides $(1+a)^{2}+a^{2} \equiv 1(\bmod 2)$. In any event $d^{*}(a)=1$ and since $d(a)$ has the same degree as $d^{*}(a)$, we must have $d(a)=1$. 
THEOREM 4.6. If $n \equiv 0$ or $8(\bmod 12)$, then $W_{n}(a)$ has no multiple roots.

Proof. Let $d(a)$ be the unique, monic, rational coefficient GCD of $W_{n}(a)$ and $W_{n}^{\prime}(a)=n W_{n-1}(a)$. As in the proof of Theorem 4.5, it follows that $d(a)$ has integer coefficients and we can write $W_{n}(a)=d(a) \cdot f(a), 2^{t-1} W_{n-1}(a)=d(a) \cdot g(a)$ where $2^{t} \| n$ and $f(a), g(a)$ have integer coefficients. Let $d(a) \equiv d^{*}(a)(\bmod 2)$ where the coefficients of $d^{*}(a)$ are 0 or 1 . It follows that $d^{*}(a)$ divides both $(1+a)^{n+2}+$ $a^{n+2}$ and $\left(1+a+a^{2}\right)\left[(1+a)^{n}+a^{n}\right](\bmod 2)$. Now, as in the proof of Theorem $4.5,1$ is the only common factor $(\bmod 2)$ of $(1+a)^{n+2}+a^{n+2}$ and $(1+a)^{n}+a^{n}$. Thus either $d^{*}(a)=1$ or $d^{*}(a)=1+a+a^{2}$. Since

$$
\begin{aligned}
(1+a)^{n+2}+a^{n+2} \equiv(1+a)^{2}(1+a)^{n}+a^{n+2} & \\
& \equiv\left(1+a^{2}+a^{4}\right)(1+a)^{n}+a^{4}(1+a)^{n}+a^{n+2} \\
& \equiv\left(1+a^{2}+a^{4}\right)\left(\sum_{k=0}^{n / 2} a^{4 k}(1+a)^{n-2 k}\right)+a^{n+2}\left(a^{n+2}+1\right)
\end{aligned}
$$

if $d^{*}(a)$ divides both $(1+a)^{n+2}+a^{n+2}$ and $1+a+a^{2}(\bmod 2)$, then $d^{*}(a)$ divides $a^{n+2}\left(a^{n+2}+1\right)(\bmod 2)$. Now

$$
\begin{aligned}
a^{n+2}+1 & \equiv(a+1)\left(1+a+a^{2}+\cdots+a^{n+1}\right) \\
& \equiv(a+1)\left(1+a+a^{2}\right)\left(1+a^{3}+\cdots+a^{n-1}\right) \text { if } n+2 \equiv 0(\bmod 3) \\
& \equiv(a+1)\left(1+a+a^{2}\right)\left(1+a^{3}+\cdots+a^{n-2}\right)+(a+1) a^{n+1} \\
& \text { if } n+2 \equiv 1(\bmod 3) \\
& \equiv(a+1)\left(1+a+a^{2}\right)\left(1+a^{3}+\cdots+a^{n-3}\right)+(a+1)^{2} a^{n}
\end{aligned}
$$$$
\text { if } n+2 \equiv 2(\bmod 3)
$$

and thus if $n \equiv 0$ or $2(\bmod 3)$ we must have $d^{*}(a)=1=d(a)$.

THEOREM 4.7. If $n \equiv 4(\bmod 12)$, then $W_{n}(a)$ has roots of multiplicity at most 2. If $W_{n}(a)$ has a multiple factor, it is of the form $a^{2}-a+c$ where $c$ is $a$ positive odd integer.

Proof. We can proceed as we do in the proofs of Theorems 4.5 and 4.6 to show that 1 is the GCD of $W_{n}(a)$ and $W_{n}^{\prime \prime}(a)$. We know from the proof of Theorem 4.6 that if $d(a)$ is the unique, monic, integer coefficient GCD of $W_{n}(a)$ and $W_{n}^{\prime}(a)$, then either $d(a)=1$ or $d(a)=a^{2}-b a+c$ where $b$ and $c$ are odd integers. Now if $W_{n}(a)=\left(a^{2}-b a+c\right)^{2} F(a)$, then since $W_{n}(a)=W_{n}(1-a)$ we have

$$
W_{n}(a)=\left[(1-a)^{2}-b(1-a)+c\right]^{2} F(1-a)=\left(a^{2}-b a+c\right) F(a) .
$$


Since $F(a)$ is square free, we have

$$
(1-a)^{2}-b(1-a)+c=a^{2}-b a+c
$$

and thus $b=1$. Since $W_{n}(a)$ has no real roots, $c$ must be positive.

It is worth noting that $W_{4}(a)=\left(1-a+a^{2}\right)^{2}$.

COROLlARY. If $n \equiv 2(\bmod 4)$, then $W_{n}(a)$ has roots of multiplicity at most 2 . If $n \equiv 3(\bmod 4)$, then $W_{n}(a)$ has roots of multiplicity at most 3 .

THEOREM 4.8. If $n \equiv 0,1,2,5,6$ or $8(\bmod 12)$, then $W_{n}(a)$ has no factor of the form $a^{2} \mid+c a+d$ where $c$ and $d$ are integers.

Proof. By the proof of Theorem 4.6, this theorem is true for $n \equiv 0$ or 8 (mod 12). In the proof of Theorem 4.6 we showed for $n$ even that $(1+a)^{n}+$ $a^{n}$ is divisible $(\bmod 2)$ by $1+a+a^{2}$ if and only if $n \equiv 0(\bmod 3)$. Now suppose $n \equiv 1$ or $5(\bmod 12)$. Then by Theorem $4.4,2^{t-1} W_{n}(a) \equiv(1+a)^{n+3}+$ $a^{n+3}(\bmod 2)$, and if $a^{2}+c a+d$ divides $2^{t-1} W_{n}(a), c, d$ odd, then $a^{2}+a+1$ divides $(1+a)^{n+3}+a^{n+3}(\bmod 2)$, which cannot happen since $n+3 \not \equiv$ $(\bmod 3)$. Since

$$
2^{t-1} W_{n}(0) \equiv 2^{t-1} W_{n}(1) \equiv 1(\bmod 2)
$$

it also follows that $a^{2}+c a+d$ does not divide $W_{n}(a)$ if $c$ or $d$ is even. The proof is similar for $n \equiv 2$ or $6(\bmod 12)$.

5. A generalization. The relationship of the numbers $W_{n}$ to the Rayleigh function $\sigma_{2 n}(-3 / 2)$ is given by (1.3). There are similar formulas involving $\sigma_{2 n}(\nu)$ where $\nu= \pm 1 / 2$ and $\nu=3 / 2$. In particular (see [12] and [7]),

$$
\begin{aligned}
\sigma_{2 n}(1 / 2) & =(-1)^{n-1} 2^{2 n-1} B_{2 n} /(2 n) !, \\
\sigma_{2 n}(-1 / 2) & =(-1)^{n} 2^{2 n-2} E_{2 n-1}(0) /(2 n-1) !, \\
\sigma_{2 n}(3 / 2) & =(-1)^{n-1} 3 \cdot 2^{2 n-1} V_{2 n} /(2 n) ! \quad(n>1),
\end{aligned}
$$

where $B_{2 n}=B_{2 n}(0)$ is the $2 n$th Bernoulli number defined by (2.18);

$$
E_{2 n-1}(0)=\frac{2\left(1-2^{2 n}\right)}{2 n} B_{2 n},
$$

the constant term of the Euler polynomial $E_{2 n-1}(a)$ defined by (2.15); and $V_{2 n}=$ $V_{2 n}(0)$ is the $2 n$th van der Pol number defined by

$$
\frac{e^{x a} x^{3} / 6}{x\left(e^{x}+1\right)-2\left(e^{x}-1\right)}=\sum_{n=0}^{\infty} V_{n}(a) \frac{x^{n}}{n !} \text {. }
$$

The Bernoulli and Euler polynomials are well known and have been studied in a 
number of papers. See [1], [2], [19, Chapter 2] and [20], for example. The present writer [6], [7] has investigated the van der Pol polynomials defined by (5.1). The van der Pol numbers were first defined and used by van der Pol [21], and an application is given in [18].

There appears to be a natural way to generalize the generating functions for the Bernoulli and Euler polynomials so as to produce the van der Pol polynomials and the polynomials of this paper, and so as to produce formulas similar to (1.3) for $\sigma_{2 n}(v), \nu$ half an odd integer. In particular, note formulas (5.13) and (5.19) of this section. Also [8] should be noted.

We shall use the following formulas [12]:

$$
\begin{gathered}
\frac{z}{2} \frac{J_{\nu+1}(z)}{J_{\nu}(z)}=\sum_{n=1}^{\infty} \sigma_{2 n}(\nu) z^{2 n}, \\
z \frac{J_{\nu-1}(z)}{J_{\nu}(z)}=2 \nu-2 \sum_{n=1}^{\infty} \sigma_{2 n}(\nu) z^{2 n},
\end{gathered}
$$

where $J_{\nu}(z)$ is the Bessel function of the first kind.

Let $y_{n}(x)$ be the Bessel polynomial defined in [14]. That is,

$$
\begin{aligned}
x^{k} y_{k}(2 / x) & =\sum_{r=0}^{k} \frac{(2 k-r) !}{r !(k-r) !} x^{r}, \\
x^{k} y_{k}(-2 / x) & =\sum_{r=0}^{k} \frac{(2 k-r) !}{r !(k-r) !}(-1)^{k-r} x^{r} .
\end{aligned}
$$

So we have

$$
\begin{gathered}
y_{0}(2 / x)=1=y_{0}(-2 / x), \\
x y_{1}(2 / x)=x+2 ; \quad x y_{1}(-2 / x)=x-2, \\
x^{2} y_{2}(2 / x)=x^{2}+6 x+12 ; \quad x^{2} y_{2}(-2 / x)=x^{2}-6 x+12 .
\end{gathered}
$$

Now for $k \geqslant 0$ define $W_{k, n}(a)$ by

$$
\frac{(-1)^{k} 2(2 k) ! e^{x a} / k !}{(-1)^{k} x^{k} y_{k}(2 / x)+e^{x} x^{k} y_{k}(-2 / x)}=\sum_{n=0}^{\infty} w_{k, n}(a) \frac{x^{n}}{n !},
$$

so for $k=0$ we get precisely (2.15) and for $k=1$ we get (1.1). That is,

If we write

$$
W_{0, n}(a)=E_{n}(a), \quad W_{1, n}(a)=W_{n}(a)
$$

$$
\sum_{r=0}^{k} b_{k, r} x^{r}=x^{k} y_{k}(-2 / x)=f_{k, 1}(x)+f_{k, 2}(x)
$$


where

$$
f_{k, 1}(x)=\sum b_{k, 2 r+1} x^{2 r+1}, \quad f_{k, 2}(x)=\sum b_{k, 2 r} x^{2 r},
$$

then we can rewrite (5.4) as

$$
\frac{(-1)^{k} 2(2 k) ! e^{x a} / k !}{f_{k, 1}(x)\left(e^{x}-1\right)+f_{k, 2}(x)\left(e^{x}+1\right)}=\sum_{n=0}^{\infty} w_{k, n}(a) \frac{x^{n}}{n !} .
$$

By replacing $x$ by $-x$ we can show, as we did in Theorem 3.1 , that

$$
W_{k, n}(a)=(-1)^{n} W_{k, n}(1-a)
$$

Also, if we define $W_{k, n}=W_{k, n}(0)$, we have

$$
W_{k, n}(a)=\sum_{r=0}^{n}\left(\begin{array}{l}
n \\
r
\end{array}\right) W_{k, r} a^{n-r}
$$

and

$$
W_{k, n}(1)=(-1)^{n} W_{k, n}
$$

Now by (5.2) and [14], we know for $k \geqslant 0$,

$\frac{-x}{4} \frac{(-1)^{k} y_{k}(2 / x)+e^{x} y_{k}(-2 / x)}{(-1)^{k+1} y_{k+1}(2 / x)+e^{x} y_{k+1}(-2 / x)}=\sum_{n=0}^{\infty}(-1)^{n} 2^{-2 n} \sigma_{2 n}\left(-k-\frac{3}{2}\right) x^{2 n}$.

Thus by (5.5), (5.6) and (5.9) we have for $k \geqslant 1$,

$$
\sum_{r=0}^{k-1}(-1)^{r} b_{k-1, r} \frac{W_{k, 2 n-r}}{(2 n-r) !}=\frac{(-1)^{n+k}(2 k) ! 2^{-2 n}}{k !} \sigma_{2 n+2}\left(-k-\frac{1}{2}\right) \text {. }
$$

Also, from (5.6) we have for $k \geqslant 1$,

$$
\sum_{r=0}^{k}(-1)^{r} b_{k, r} \frac{W_{k, 2 n-r}}{(2 n-r) !}=0 .
$$

A recurrence formula, which can be derived from (5.6), is $\frac{(-1)^{k} 2(2 k) !}{k !} a^{n}$

$$
\begin{aligned}
= & \sum_{r=0}^{n} \frac{(-1)^{n-r} n ! b_{k, n-r}}{r !} W_{k, r}(a) \\
& +\sum_{r=0}^{n}\left(\left(\begin{array}{l}
n \\
r
\end{array}\right) b_{k, 0}+\sum_{s=1}^{n=r}\left(\begin{array}{l}
n \\
r
\end{array}\right)(n-r) \cdots(n-r-s+1) b_{k, s}\right) W_{k, r}(a) .
\end{aligned}
$$

For example, we have

$$
\frac{24 e^{x a}}{-6 x\left(e^{x}-1\right)+\left(x^{2}+12\right)\left(e^{x}+1\right)}=\sum_{n=0}^{\infty} w_{2, n}(a) \frac{x^{n}}{n !}
$$


where

$$
\frac{12 W_{2,2 n}}{(2 n !)}+\frac{6 W_{2,2 n-1}}{(2 n-1) !}+\frac{W_{2,2 n-2}}{(2 n-2) !}=0
$$

and

$$
\frac{-2 W_{2,2 n}}{(2 n) !}-\frac{W_{2,2 n-1}}{(2 n-1) !}=\frac{(-1)^{n} 12}{2^{2 n}} \sigma_{2 n+2}(-5 / 2)
$$

Thus we see that

$$
\sigma_{2 n+2}(-5 / 2)=\frac{(-1)^{n} 2^{2 n-3}}{9(2 n-2) !} W_{2,2 n-2}
$$

A recurrence formula for $W_{2, n}(a)$ is

$$
\begin{aligned}
24 a^{n}= & 12 W_{2, n}(a)+6 n W_{2, n-1}(a)+n(n-1) W_{2, n-2}(a) \\
& +\sum_{r=0}^{n}\left(\begin{array}{l}
n \\
r
\end{array}\right)[12+(n-r)(n-r-7)] W_{2, r}(a)
\end{aligned}
$$

so that

$$
W_{2,0}(a)=1, \quad W_{2,1}(a)=a-1 / 2, \quad W_{2,2}(a)=a^{2}-a+1 / 3 .
$$

We can proceed in the same way to generalize the generating function for the Bernoulli polynomials. In this case we define $V_{k, n}(a)$ by means of

$$
\frac{k ! x^{2 k+1} e^{x a} /(2 k+1) !}{(-1)^{k-1} x^{k} y_{k}(2 / x)+e^{x} x^{k} y_{k}(-2 / x)}=\sum_{n=0}^{\infty} V_{k, n}(a) \frac{x^{n}}{n !}
$$

This can be written

$$
\frac{k ! x^{2 k+1} e^{x a} /(2 k+1) !}{f_{k, 1}(x)\left(e^{x}+1\right)+f_{k, 2}(x)\left(e^{x}-1\right)}=\sum_{n=0}^{\infty} V_{k, n}(a) \frac{x^{n}}{n !} .
$$

Thus we have

$$
V_{0, n}(a)=B_{n}(a), \quad V_{1, n}(a)=V_{n}(a)
$$

Generally, we have

$$
V_{k, n}(a)=(-1)^{n} V_{k, n}(1-a)
$$

and if $V_{k, n}(0)=V_{k, n}$, we have

$$
V_{k, n}(a)=\sum_{r=0}^{n}\left(\begin{array}{l}
n \\
r
\end{array}\right) V_{k, r} x^{n-r}, \quad V_{k, n}(1)=(-1)^{n} V_{k, n} .
$$

Proceeding as before, but using (5.3), we have for $k \geqslant 1, n>k$, 


$$
\begin{aligned}
& \sum_{r=0}^{k-1}(-1)^{r-1} b_{k-1, r} \frac{V_{k, 2 n-r+1}}{(2 n-r+1) !} \\
& \quad=\frac{k !}{(2 k+1) !}(-1)^{n-k} 2^{-1-2 n+2 k} \sigma_{2 n-2 k+2}\left(k+\frac{1}{2}\right),
\end{aligned}
$$

and for $n>k$,

$$
\sum_{r=0}^{k}(-1)^{r} b_{k, r} \frac{V_{k, 2 n+1-r}}{(2 n+1-r) !}=0 .
$$

A recurrence formula is

$$
\begin{aligned}
\sum_{r=0}^{n}\left[\left(\begin{array}{l}
n \\
r
\end{array}\right) b_{k, 0}\right. & \left.+\sum_{s=1}^{n-r}\left(\begin{array}{l}
n \\
r
\end{array}\right)(n-r) \cdots(n-r-s+1) b_{k, s}\right] V_{k, r}(a) \\
& +\sum_{r=0}^{n} \frac{(-1)^{n-r+1} n ! b_{k, n-r}}{r !} V_{k, r}(a) \\
& = \begin{cases}0 & \text { if } n<2 k+1, \\
\frac{k ! n !}{(2 k+1) !} \frac{a^{n-2 k-1}}{(n-2 k-1) !} & \text { if } n \geqslant 2 k+1 .\end{cases}
\end{aligned}
$$

For example

$$
\frac{e^{x a} x^{5} / 60}{-6 x\left(e^{x}+1\right)+\left(x^{2}+12\right)\left(e^{x}-1\right)}=\sum_{n=0}^{\infty} V_{2, n}(a) \frac{x^{n}}{n !}
$$

and by (5.16) and (5.17) we have for $n>1$

$$
\sigma_{2 n}(5 / 2)=\frac{(-1)^{n} 2^{2 n} \cdot 5}{(2 n+1) !} V_{2,2 n+1}
$$

The recurrence formula is

$$
\begin{aligned}
\sum_{r=0}^{n}\left(\begin{array}{l}
n \\
r
\end{array}\right)[12+(n-r) & (n-r-7)] V_{2, r}(a)-n(n-1) V_{2, n-2}(a) \\
& -6 n V_{2, n-1}(a)-12 V_{2, n}(a) \\
= & \begin{cases}0 & \text { if } n<5, \\
\frac{n !}{60(n-5) !} a^{n-5} & \text { if } n \geqslant 5 .\end{cases}
\end{aligned}
$$

The first three polynomials are

$$
V_{2,0}(a)=1, \quad V_{2,1}(a)=a-1 / 2, \quad V_{2,2}(a)=a^{2}-a+3 / 14 .
$$


Note that by (5.5) and (5.14) we always have $W_{k, 0}=1=V_{k, 0}$.

\section{REFERENCES}

1. J. Brillhart, On the Euler and Bernoulli polynomials, J. Reine Angew. Math 234 (1969), 45-64. MR 39 \#4117.

2. - Some modular results on the Euler and Bernoulli polynomials, Acta Arith. 21 (1972), 173-181. MR 46 \#3433.

3. L. Carlitz, A sequence of integers related to the Bessel functions, Proc. Amer. Math. Soc. 14 (1963), 1-9. MR 29 \#3425.

4. - Recurrences for the Rayleigh functions, Duke Math. J. 34 (1967), 581-590. MR $35 \# 5670$.

5. F. T. Howard, $A$ property of a class of nonlinear difference equations, Proc. Amer. Math. Soc. 38 (1973), 15-21. MR 46 \#8954.

6. - Properties of the van der Pol numbers and polynomials, J. Reine Angew. Math. 260 (1973), 35-46. MR 47 \#6603.

7. The van der Pol numbers and a related sequence of rational numbers, Math. Nachr. 42 (1969), 80-102. MR 41 \#3385.

8. - Generalized van der Pol numbers, Math. Nachr. 44 (1970), 181-191. MR $45 \# 8600$.

9. G. S. Kazantzidis, On a congruence and on a practical method for finding the highest power of a prime $p$ which divides the binomial coefficient $\left(\begin{array}{l}A \\ B\end{array}\right)$, Bull. Soc. Math. Grèce 6 II (1965), fasc. 2, 358-360. (Grêk) MR 34 \#7440.

10. N. Kishore, $A$ class of formulas for the Rayleigh function, Duke Math. J. 34 (1967), 573-580. MR 35 \#5669.

11. A structure of the Rayleigh polynomial, Duke Math. J. 31 (1964), 513-518. MR $29 \# 1370$.

12. - The Rayleigh function, Proc. Amer. Math. Soc. 14 (1963), 527-533. MR 27 \#1633.

13. - The Rayleigh polynomial, Proc. Amer. Math. Soc. 15 (1964), 911 917. MR 29 \#6079.

14. H. L. Krall and O. Frink, $A$ new class of orthogonal polynomials: the Bessel polynomials, Trans. Amer. Math. Soc. 65 (1949), 100-115. MR 10, 453. $405-407$.

15. D. H. Lehmer, Zeros of the Bessel function $J_{\nu}(x)$, Math. Comp. 1 (1943-1945),

16. N. Liron, A recurrence concerning Rayleigh functions, SIAM J. Math. Anal. 2 (1971), 496-499. MR 46 \#5696.

17. Infinite sums of roots for a class of transcendental equations and Bessel functions of order one-half, Math. Comp. 25 (1971), 769-781. MR 46 \#3858.

18. L. Lorch, The limits of indetermination for Riemann summation in terms of Bessel functions, Colloq. Math. 15 (1966), 313-318. MR 34 \#6377. 1954.

19. N. E. Nörlund, Vorlesungen über Differenzenrechnung, Springer-Verlag, New York,

20. R. Spira, The nonvanishing of the Bernoulli polynomials in the critical strip, Proc. Amer. Math. Soc. 17 (1966), 1466-1467. MR 34 \#2967.

21. B. van der Pol, Smoothing and "unsmoothing", Probability and Related Topics in Physical Sciences, New York, 1957, pp. 223-235.

22. G. N. Watson, A treatise on the theory of Bessel functions, Cambridge Univ. Press, New York, 1962.

DEPARTMENT OF MATHEMATICS, WAKE FOREST UNIVERSITY, WINSTONSALEM, NORTH CAROLINA 27109 